\title{
YouTube e user generated content: $O$ seu valor e a sua utilidade no setor do turismo em Portugal
}

\section{YouTube and user generated content: Its value and utility in the tourism sector in Portugal}

\author{
Carolina Afonso
}

ISEG, Universidade de Lisboa, Portugal

carolinaafonso@iseg.ulisboa.pt

Patrícia Ceia

ISEG, Universidade de Lisboa, Portugal

patricia.a.s.ceia@gmail.com

\section{Resumo}

\begin{abstract}
Os consumidores recorrem ao user generated content (UGC) para tomar decisões, nomeadamente escolhas relacionadas com o turismo, como viagens, acomodação e restaurantes. O objetivo deste estudo é compreender a relação existente entre o valor funcional e a utilidade percebida pelos consumidores em relação ao UGC, considerando o contexto do turismo no YouTube. O estudo é quantitativo, tendo-se obtido 748 respostas ao questionário online dirigido a utilizadores do YouTube em Portugal. Através da análise de dados multivariada verificou-se que quanto maior a perceção do valor funcional do UGC, maior a utilidade percebida destes conteúdos. O estudo contribui para um maior conhecimento do uso do UGC, sugerindo que empresas na área do turismo apostem na criação de conteúdo útil para os consumidores.
\end{abstract}

Palavras-chave: User generated content; valor funcional; utilidade percebida; YouTube.

\begin{abstract}
Consumers are turning to user generated content (UGC) to make decisions, including tourismrelated choices such as travel, accommodation and restaurants. The objective of this study is to understand the relationship between the functional value and the perceived utility of the UGC by consumers, considering the context of tourism in YouTube. The study is quantitative and in total 748 responses were obtained through an online questionnaire addressed to YouTube users in Portugal. Through the analysis of multivariate data it was verified that the greater the perception of the functional value of the UGC, the greater the perceived utility of these contents. The study contributes to a greater knowledge of the use of UGC, suggesting that companies in the tourism area should engage in the creation of useful content for consumers.
\end{abstract}

Keywords: User generated content; functional value; perceived utility; YouTube.

\section{Introdução}

A evolução da World Wide Web (www ou web) coloca novos desafios à gestão, sobretudo ao marketing. A web 2.0 trouxe consigo a criação de redes de utilizadores, sejam eles empresas ou consumidores (Constantinides, 2009; Constantinides \& Fountain, 2008; Levy, 2009). A Dos Algarves: A Multidisciplinary e-Journal, $32-2018$. ISBN 2182-5580 @ ESGHT - University of the Algarve, Portugal.

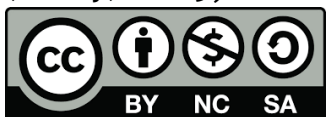

To cite this article: Afonso, C. \& Ceia, P. (2018). YouTube e user generated content: $O$ seu valor e a sua utilidade no setor do turismo em Portugal. Dos Algarves: A Multidisciplinary e-Journal, 32, 3-19. doi: 10.18089/DAMeJ.2018.32.1 
evidência de diversas plataformas que surgiram com o advento da web 2.0 contribuem para que os fluxos de conhecimento e informação se tornem mais dinâmicos e interativos (Constantinides \& Fountain, 2008; O'Reilly \& Battelle, 2009), levando ao aparecimento do conceito de redes sociais, como o YouTube, e ao fenómeno do user generated content (UGC), isto é, conteúdo gerado e distribuído pelo próprio utilizador. Este fenómeno veio originar uma nova dinâmica na fase de procura de informação, durante o processo de tomada de decisão de compra pelos consumidores (Cheong \& Morrison, 2008; Court, Elzinga, Mulder \& Vetvik, 2009; Lecinski, 2011). Esta nova dinâmica reflete-se em contextos como o turismo (Reino \& Hay, 2011), uma vez que os consumidores, enquanto turistas, também procuram diversas informações no meio online, no momento em que planeiam viagens.

O YouTube apresenta-se como uma rede social de partilha de conteúdo através de vídeos (Pikas \& Sorrentino, 2014; Shao, 2009; Snelson, 2011). Esta plataforma da web 2.0, para além da partilha de vídeos, permite também a interação entre utilizadores por comentários, "gostos" ou partilhas dos mesmos (Shao, 2009). Hoje em dia, o YouTube tem-se afirmado cada vez mais (Shao, 2009; Snelson, 2011), ultrapassando os mil milhões de utilizadores (YouTube, 2016b), sendo uma das plataformas de UGC mais relevantes, incluindo no setor turístico.

O UGC é uma característica da web 2.0 (Constantinides \& Fountain, 2008) e diz respeito ao conteúdo que é criado e distribuído online por qualquer indivíduo, através de vários formatos, incluindo o vídeo (Daugherty, 2008; Wunsch-Vincent \& Vickery, 2007) e que pode fornecer informações diversas aos consumidores, tal como opiniões ou experiências de utilização (Cummins, Peltier, Schibrowsky \& Nill, 2014). Atendendo ao contexto do setor do turismo, são exemplos de UGC roteiros turísticos ou reviews acerca de locais emblemáticos das cidades partilhados por utilizadores na internet.

Neste sentido, considera-se relevante abordar mais profundamente esta temática, devido não só à atualidade do UGC, como também à sua importância no quotidiano dos consumidores e ao grande número de utilizadores do YouTube. Pretende-se, essencialmente, analisar o impacto que o valor funcional do UGC partilhado no YouTube tem na utilidade percebida pelos consumidores portugueses, atendendo ao contexto do turismo no geral. Como tal, o problema de investigação deste estudo passa por compreender qual o impacto que o valor funcional do UGC partilhado no YouTube tem na utilidade percebida pelos utilizadores em relação a esse conteúdo.

\section{Revisão de literatura}

\subsection{A web 2.0}

A web 2.0 trata-se de "uma coleção de aplicações online de fonte aberta, interativas e controladas pelo utilizador que expande as experiências, o conhecimento e o poder de mercado dos utilizadores como participantes de processos de negócio e sociais" (Constantinides \& Fountain, 2008: 232-233). Estas aplicações/plataformas são o suporte para as redes de utilizadores criadas, que permitem a existência de fluxos de conteúdo, informação e conhecimento (Constantinides \& Fountain, 2008), fluxos esses que se movem entre criadores e consumidores (Cormode \& Krishnamurthy, 2008). 
$\mathrm{Na}$ perspetiva dos consumidores, a web 2.0 oferece novas possibilidades, principalmente como fonte de informação e meio de comunicação diária. Para além disto, importa salientar que a web 2.0 conduziu ainda a alterações nas necessidades, nas perceções de valor e no comportamento de compra dos consumidores (Constantinides \& Fountain, 2008). Os consumidores, ao nível das suas preferências e decisões, baseiam-se cada vez mais em informações provenientes de fontes não controláveis pelos marketers, cujo conteúdo é criado pelos próprios utilizadores (UGC), de forma ativa, no meio online, nomeadamente em blogs, redes sociais e fóruns (Constantinides, 2009; Constantinides \& Fountain, 2008). Neste sentido, e uma vez que o conteúdo online é de fácil acesso e poderá ser duradouro, estes utilizadores podem até ser vistos como líderes de opinião e ter influência na decisão de compra de outros consumidores (Cheong \& Morrison, 2008).

\subsection{A consumer journey}

O processo de decisão de compra sofreu alterações (Lecinski, 2011). A forma como os consumidores se comprometem com as marcas e tomam as suas decisões de compra mudou devido às transformações ocorridas no ambiente digital, nomeadamente ao nível da web 2.0, das tecnologias, das plataformas, dos dispositivos móveis e dos canais sociais (Edelman, 2010; Lieb, Owyang, Gropman \& Silva, 2012). De acordo com o estudo da Google, em Portugal, $83,0 \%$ dos inquiridos utiliza diariamente a internet nos computadores, smartphones e tablets, para assuntos pessoais (Google, 2016a), e 49,0\% utilizam o meio online para aprender mais sobre produtos que compram, ainda que utilizem ambas os meios online e offline para obter informações antes de efetuarem uma compra (Google, 2015; Google, 2016a). Inclusive, segundo outro estudo da Google, a pesquisa online influência bastante a compra (Google, 2016c). Verifica-se a emergência de canais digitais que, por sua vez, conduziram a um consumidor mais informado, processo este designado por consumer empowerment (Court et al., 2009). Este consumidor é caracterizado pela experiência, pelo dinamismo, pela posse de informações úteis que procuram ativamente e constantemente antes de efetuarem uma compra, e ainda por terem à disposição vários tipos dispositivos que o auxiliam na tomada de decisão (Court et al., 2009; Lecinski, 2011; Lieb et al., 2012). Ao nível da procura de informações, os consumidores recorrem a várias fontes, nomeadamente reviews sobre os produtos ou lugares, ratings na internet e nas redes sociais, passa-a-palavra de familiares e amigos, websites das marcas, textos, imagens, vídeos (este é um dos formatos que começou a ser mais utilizado), interações que ocorrem no interior das lojas e experiências de compra anteriores (Court et al., 2009; Lecinski, 2011). É de salientar também o facto de os consumidores recorrerem a informação gerada por outros acerca dos produtos antes de procederem a uma compra, principalmente se esta for de elevado envolvimento (Cheong \& Morrison, 2008). Estas alterações ocorreram também no setor do turismo, em que os próprios consumidores utilizam informações do meio online, como reviews sobre viagens ou hotéis, ou passa-a-palavra para tomarem as suas decisões (Ye, Law, Gu \& Chen, 2011), tendo estas informações um grande impacto nas suas perceções da marca (Barreda \& Bilgihan, 2013).

Foi a partir deste contexto que surgiu uma nova abordagem mais sofisticada que a anterior, designada por consumer decision journey (Court et al., 2009; Edelman \& Singer, 2015a; Lecinski, 2011) e que diz respeito à forma como os consumidores se comportam e 
interagem, tendo em vista um determinado objetivo (Edelman \& Singer, 2015a). Trata-se de um ciclo composto por quatro etapas: consideração inicial, avaliação ativa, compra e póscompra. É no momento da avaliação ativa que os consumidores adicionam ou retiram marcas do conjunto inicial que consideraram para a compra, conforme a avaliação que fazem delas (Court et al., 2009). Neste momento, também designado por Zero Moment Of Truth (ZMOT) pela Google, as mensagens informativas dão lugar à conversação e à troca de informações entre consumidores, empresas/marcas e outros intervenientes. Há também a aprendizagem sobre os produtos e são realizadas escolhas, em tempo real e em qualquer lugar, por conseguinte, é um momento de elevado impacto para os consumidores, no qual as empresas/marcas devem estar presentes de forma marcante e influente (Lecinski, 2011). Neste sentido, a procura de informação sobre as marcas e os produtos é essencial. Adicionalmente, de acordo com um estudo mais recente de Edelman e Singer (2015b), verifica-se já uma evolução da consumer decision journey, em que de alguma forma, as empresas conseguem moldar e otimizar os ciclos de decisão dos consumidores, levando-os a saltar as duas primeiras etapas, focando-se essencialmente em acelerar o processo de fidelização dos consumidores às marcas.

\subsection{O papel do vídeo e do YouTube}

Atualmente, o YouTube já ultrapassa os mil milhões de utilizadores a nível mundial, apresentando um crescimento significativo ao nível do tempo de visualização dos vídeos, assim como do número de pessoas que diariamente utilizam esta rede social (YouTube, 2016b), principalmente através de dispositivos móveis (Google, 2016a).

Fazendo a ligação ao momento de avaliação ativa ou ZMOT, verifica-se que grande parte do conteúdo disponível para os utilizadores é essencialmente visual, como é o exemplo do vídeo. Os consumidores procuram na sua maioria vídeos relacionados com demonstrações, how to's e conselhos profissionais ou mais específicos sobre determinados temas (Lecinski, 2011). Também são partilhados inúmeros vídeos relacionados com o turismo, como demonstram Reino e Hay (2011) no seu estudo acerca do uso do YouTube como ferramenta de marketing. Trata-se de um formato de fácil partilha e acesso através da internet e, por conseguinte, com benefícios para chamar a atenção dos visualizadores para determinado conteúdo (Lecinski, 2011). Adicionalmente, segundo estudos realizados pela Marktest e pela Google, respetivamente $87,0 \%$ dos inquiridos assiste a conteúdos em vídeos nas redes sociais (Marktest Consulting, 2016) e 34,0\% dos inquiridos veem vídeos online diariamente, através de computadores, smarpthones e tablets (Google, 2016b), o que também é um indicador da importância deste formato.

Importa ainda referir que o vídeo é um formato que pode ser utilizado não só pelos consumidores em geral que tenham conteúdo a partilhar, como também pelas próprias marcas; inclusive verifica-se que é um formato com tendência crescente na sua importância e utilização no mundo digital e online, apesar de ser pouco usado e explorado por empresas e marketers em Portugal (GBSN Research, 2016). No setor do turismo, apesar de o UGC predominar, há empresas a criar conteúdo em vídeo, nomeadamente empresas de transportes e de hotelaria (Reino \& Hay, 2011).

No que diz respeito às plataformas de visualização de vídeos, atualmente, destaca-se o YouTube (GBSN Research, 2016). Este, produto da Google, foi criado em maio de 2005 
(YouTube, 2016a) e, desde então, tem-se tornado cada vez mais no meio online (Shao, 2009; Snelson, 2011). Na sua essência, o YouTube é uma plataforma de partilha de vídeos online, sendo este também o propósito para o qual foi criado (Snelson, 2011) e, para além disso, é visto como um social networking site (SNS), uma das redes sociais mais populares (Pikas \& Sorrentino, 2014).

Importa referir que, para os utilizadores que criam e partilham os vídeos, esta é uma plataforma de UGC fácil de utilizar, pois os passos necessários para colocar um vídeo online no YouTube são simples e relativamente rápidos de executar. No que diz respeito aos motivos de partilha de conteúdo destacam-se a auto-expressão e a auto-realização (Shao, 2009). Por outro lado, ao nível do conteúdo que é possível visualizar nesta plataforma online, os vídeos partilhados têm temas bastante variados, tais com filmes, música, notícias, desporto, comédia, entre outros (Shao, 2009; GBSN Research, 2016).

Vários estudos referem a importância ou exemplificam o YouTube como uma das plataformas principais de UGC (Cheong \& Morrison, 2008; Kim, Jin, Kim \& Shin, 2012; Mir \& Rehman, 2013; Paek, Hove, Jeong \& Kim, 2011; Reino \& Hay, 2011; Smith, Fischer \& Yongjian, 2012). De acordo com um estudo de Cheong e Morrison (2008), os inquiridos utilizam o YouTube mais de três vezes por semana, principalmente por divertimento e entretenimento ou porque lhes foram enviados hyperlinks de vídeos por amigos. Segundo Shao (2009), os consumidores procuram satisfazer necessidades de informação e também de entretenimento no UGC.

Em Portugal, de acordo com dados recolhidos em 2016, 43,0\% dos utilizadores de redes sociais possuem conta no YouTube, número que aumentou face ao ano anterior (Marktest Consulting, 2015; Marktest Consulting, 2016). Observa-se também que o YouTube é o terceiro site com maior número de utilizadores e visualizações, com cerca de dois milhões e meio de utilizadores por semana (Havas Media Group, 2016; Marktest, 2016). No âmbito empresarial, segundo um estudo realizado pela GBSN Research (2016), apesar de 62,0\% dos marketers inquiridos não utilizarem esta rede social, outros referem que a utilizam principalmente com os objetivos de informar os consumidores e de criar ou fortalecer a ligação emocional que estabelecem com eles. As marcas que participaram no estudo indicam também que a sua produção de conteúdo no meio digital é crescente $(86,7 \%)$, integrando as redes sociais nas suas estratégias de marketing (98,3\%). Estes motivos demonstram que o YouTube é uma plataforma de excelência, onde as marcas se podem dar a conhecer e partilhar conteúdo útil, de forma dinâmica e interativa com o seu público-alvo. Nomeadamente no setor do turismo, trata-se de uma plataforma que permite aos utilizadores obterem informações, verem reviews ou guias de viagem, terem conhecimento de experiências de outras pessoas nas várias atividades relacionadas com o destino que desejam conhecer, sendo que este conteúdo é considerado bastante confiável e influente (Reino \& Hay, 2011).

\section{4. $O$ UCG, o valor funcional e a utilidade percebida}

O UGC é caracterizado por três aspetos principais: o conteúdo é publicado online, num website ou numa rede social acessível a um conjunto ou a todos os indivíduos, atendendo a um determinado contexto; necessita de um esforço criativo dos autores, na medida em que estes devem contribuir com o seu próprio valor para o conteúdo que publicam; e deve ser feito fora do âmbito profissional dos utilizadores, apesar de esta característica ser de difícil 
observância, pelo facto de muitos utilizadores serem remunerados precisamente pelo conteúdo que criam (Wunsch-Vincent \& Vickery, 2007).

$\mathrm{O}$ valor funcional diz respeito à existência de atributos funcionais, utilitários e físicos relevantes (Sheth, Newman \& Gross, 1991). No caso específico do UGC, relaciona-se com o facto de os consumidores verem satisfeitos os seus desejos de informação relativos a objetivos e necessidades práticas dos produtos, nomeadamente a conveniência, a disponibilidade, a facilidade de uso, o preço ou a durabilidade (Kim et al., 2012; Sheth et al., 1991). Tendo como base o setor do turismo, o valor funcional do UGC diz respeito, por exemplo, ao facto de um consumidor ter acesso a um determinado conteúdo e obter as informações que necessita sobre a cidade para onde irá viajar, o preço médio e características dos hotéis onde poderá pernoitar ou ainda os locais mais emblemáticos a visitar.

Kim et al. (2012) consideram que o valor funcional do UGC tem um impacto positivo na utilidade do mesmo conteúdo para os consumidores, ou seja, influencia os benefícios e a experiência que os consumidores podem obter a partir da utilização desse conteúdo. A utilidade percebida pelo consumidor, segundo Pinho e Soares (2011), atendendo a um contexto tecnológico, baseia-se no facto dos consumidores acreditaram que a adoção de uma determina tecnologia poderá levá-los a um melhor desempenho. Em concordância estão também Davis, Bagozzi e Warshaw (1989) e Mir e Rehman (2013). Estes autores expõem o conceito de utilidade percebida como o facto de uma aplicação ou sistema ser utilizado por um indivíduo ter uma grande probabilidade de melhorar o desempenho deste num determinado contexto. Tendo em consideração o contexto turístico, a utilidade do UGC está relacionada com o facto de um consumidor considerar que a visualização de determinados conteúdos em vídeo lhe irá ser útil e fará com que consiga planear melhor a sua viagem.

Considerando os estudos destes autores, podem observar-se evidências de uma relação existente entre o valor funcional do UGC e a utilidade percebida do UGC para os consumidores. Neste sentido, propõe-se testar a seguinte hipótese de investigação:

- H1 - Existe uma relação positiva entre o valor funcional do UGC para os consumidores e a sua utilidade percebida.

\section{Metodologia}

\subsection{Tipo de estudo e amostra}

O propósito da presente investigação é explanatório, uma vez que se pretende compreender uma relação entre várias variáveis (Saunders, Lewis \& Thornhill, 2009), nomeadamente acerca do fenómeno do UGC no YouTube.

Seguiu-se uma estratégia de inquérito, sendo que esta permite a recolha de uma grande quantidade de dados a um custo relativamente baixo, assim como uma fácil comparação dos mesmos (Malhotra \& Birks, 2006; Saunders et al., 2009).

A população do estudo é constituída por utilizadores da plataforma online YouTube (incluem-se quer os utilizadores que visualizam o conteúdo em vídeo, quer os próprios YouTubers) em Portugal. A amostra, sendo um subgrupo da população, inclui indivíduos com as mesmas características desta última (Malhotra \& Birks, 2006; Saunders et al., 2009).

Neste estudo utilizou-se, assim, uma amostragem não probabilística por conveniência. 


\subsection{Recolha de dados}

Para a recolha de dados da pesquisa utilizou-se o processo de método único, optando por um estudo quantitativo (Saunders et al., 2009).

Neste sentido, o método utilizado na recolha de dados foi o questionário estruturado, auto-administrado pelos respondentes, mediado pela internet, permitindo recolher dados de uma amostra relativamente grande (Malhotra \& Birks, 2006; Saunders et al., 2009). O questionário foi partilhado online, na plataforma Qualtrics, através de vários meios, entre os quais o Facebook, o e-mail, o YouTube e os canais institucionais do ISEG (Instituto Superior de Economia e Gestão). A partilha foi feita junto de familiares, amigos, conhecidos YouTubers. A recolha de dados através do questionário foi efetuada entre 1 de julho e 15 de agosto de 2016.

O estudo também requereu a recolha de dados secundários obtidos em bases de dados bibliográficas e noutros websites. Esta recolha teve como foco a informação relativa à web, à consumer journey, ao papel do vídeo, ao YouTube e ao UGC, considerando o turismo como contexto base.

\subsection{Questionário e escalas de medida}

No que diz respeito à recolha de dados para este estudo, foi realizado um questionário (ver Anexo 1), em que foi colocado um conjunto de questões aos inquiridos que afirmaram ser utilizadores do YouTube. Estas questões foram mensuradas em escalas de concordância tipo Likert (Malhotra \& Birks, 2006), de sete pontos (entre 1- Discordo Totalmente e 7 - Concordo Totalmente), tiveram por base as escalas de valor funcional de Zainuddin, Russell-Bennett e Previte (2013) e utilidade percebida de Mir e Reham (2013).

\section{Análise de resultados}

\subsection{Caracterização da amostra}

A amostra do presente estudo (ver Anexo 2) é constituída por 748 inquiridos, que afirmaram ser utilizadores do YouTube, sendo $61,9 \%$ do sexo feminino e $38,1 \%$ do sexo masculino. A maioria dos respondentes são jovens com menos de 35 anos (90,6\%), sendo a faixa etária mais significante pertence às idades compreendidas entre os 20 e os 24 anos (36,8\%). Ao nível da ocupação, 66,0\% são estudantes, $8,7 \%$ estudam e trabalham em simultâneo, 17,2\% trabalham por conta de outrem e apenas $4,4 \%$ estão desempregados. Relativamente às habilitações académicas, a maior parte dos inquiridos tem uma licenciatura (30,2\%) e apenas $11,4 \%$ das pessoas possuem grau superior a este. No que diz respeito à distribuição dos elementos da amostra em Portugal, cerca de metade reside em Lisboa (52,7\%), seguindo-se os distritos do Porto (12,0\%) e Setúbal (9,6\%). Já quanto ao rendimento do agregado familiar dos respondentes, apesar de nem todos se terem pronunciado sobre este assunto, a maioria afirma que o rendimento se situa entre $1001 €$ e $1500 €(16,2 \%)$, referindo também que é possível viver com o rendimento que recebem $(47,7 \%)$. Quanto ao nível de horas diárias de utilização de internet e do YouTube, em média, a maioria dos inquiridos referiu passar mais de seis horas na internet $(27,1 \%)$ e entre uma a duras horas no YouTube $(28,0 \%)$. 
Por fim, de forma a conhecer um pouco melhor sobre a amostra do estudo, tentou-se compreender os motivos pelos quais os respondentes utilizam o YouTube e quais as categorias de vídeo que mais visualizam. Em média, verificou-se que os inquiridos utilizam o YouTube principalmente por entretenimento $(\overline{\mathrm{X}} 1=6,54)$, para passar o tempo $(\overline{\mathrm{X} 2}=6,02)$ e para procurar informação ( $\overline{\mathrm{X}} 3=5,80)$ e que as categorias de vídeo que veem com maior frequência dizem respeito a vídeos sobre música $(\overline{\mathrm{X} 4}=5,96)$, comédia $(\overline{\mathrm{X} 5}=4,63)$ e how to's e DIY's (Do It Youself - $\overline{\mathrm{X}} \overline{6}=3,97$ ).

\subsection{Análise fatorial}

Realizou-se uma análise fatorial (Marôco, 2014) com o objetivo de analisar a estrutura das variáveis do estudo - as estatísticas descritivas destas variáveis encontram-se no Anexo 3. Mais especificamente, o método de extração de fatores utilizado foi a medida de adequação da amostragem de Kaiser-Meyer-Olkin (KMO).

Tabela 1. KMO e teste de Bartlett

\begin{tabular}{c|c|c}
\hline Variáveis & Valor Funcional & Utilidade \\
\hline KMO & 0,830 & 0,867 \\
Variância Total Explicada (\%) & 73,463 & 64,279 \\
Teste de Esfericidade de Bartlett & $X^{2}(6)=1555,013$ & $X^{2}(15)=2421,660$ \\
& valor- $p=0,000$ & valor- $p=0,000$ \\
\hline
\end{tabular}

De acordo com os resultados da Tabela 1, verifica-se que a adequabilidade da análise fatorial das variáveis é boa, assim como a percentagem de variância explicada é relativamente elevada. Adicionalmente, através do teste de Esfericidade de Bartlett, observa-se que ambas as variáveis estão correlacionadas significativamente.

\subsection{Análise de fiabilidade e consistência interna}

Para analisar a fiabilidade dos índices sintéticos recorreu-se ao cálculo do Alfa de Cronbach (Marôco, 2014). O Alfa de Cronbach das variáveis em estudo é de 0,870 (valor funcional) e 0,885 (utilidade percebida), o que significa que os itens apresentam uma boa fiabilidade e a sua consistência interna é muito boa.

\subsection{Validação da hipótese de investigação}

Os procedimentos seguidos e as análises realizadas para a validação da hipótese de investigação deste estudo tiveram como referência Malhotra e Birks (2006) e Marôco (2014). De forma a validar a hipótese de investigação, recorreu-se a uma regressão linear simples, na 
qual a variável dependente é a utilidade percebida e a variável independente é o valor funcional.

Primeiramente, realizou-se o teste de Kolmogorov-Smirnov (K-S) para testar a normalidade das variáveis. Através da análise dos resultados verifica-se que as variáveis valor funcional (K-SValor Funcional $=0,096$, valor $-p=0,000, \alpha=0,05$ ) e utilidade percebida (K$S_{U t i l i d a d e}=0,071$, valor- $\left.p=0,000, \alpha=0,05\right)$ não seguem uma distribuição Normal, visto a hipótese nula deste teste ser rejeitada. No entanto, pelo facto da amostra ser considerada de grande dimensão $(n=748>30)$, recorrendo ao Teorema do Limite Central (TLC) é possível pressupor que estas variáveis seguem uma distribuição Normal. Por conseguinte, é possível realizar a regressão. De seguida, testou-se a validade dos pressupostos da regressão, tendo-se concluído que todos são válidos.

Quanto ao modelo da regressão, de acordo com a Tabela 2 e pela análise do coeficiente de determinação ajustado ( $R^{2}$ ajustado) verifica-se que 52,0\% da variação total da utilidade é explicada pela variação da variável valor funcional. Através do teste $F(F(1)=599,076$, valor$p=0,000, a=0,05)$, pode afirmar-se que o modelo é significativo e válido, visto rejeitar-se a hipótese nula deste teste. $O$ erro padrão da estimativa $(S=0,68618)$ é baixo, o que indica um bom ajuste entre os valores observados da amostra e os valores estimados pelo modelo. Verifica-se que o valor funcional tem impacto positivo e significativo na utilidade percebida $(B=0,667 ; t=14,072 ;$ valor- $p=0,000 ; \alpha=0,05)$, validando-se a hipótese em estudo.

Tabela 2. Regressão linear simples (H1)

\begin{tabular}{c|c|c|c}
\cline { 2 - 4 } & \multicolumn{3}{c}{ Variável dependente: Utilidade percebida } \\
\cline { 2 - 4 } & $\begin{array}{c}\text { Coeficiente } \\
\text { padronizado }\end{array}$ & $\mathrm{t}$ & Valor $-p$ \\
\hline Valor funcional & 0,667 & 14,072 & 0,000 \\
$\mathrm{~F}(1)=599,076$ & & & \\
$\mathrm{R}^{2}=0,520$ & & & \\
\hline
\end{tabular}

\section{Conclusões, limitações e pesquisa futura}

O problema de investigação deste estudo é compreender a relação existente entre o valor funcional do UGC partilhado no YouTube e a utilidade percebida pelos consumidores em relação a esse conteúdo, em Portugal, tendo em consideração o setor do turismo.

Verificou-se, através da análise de uma regressão linear simples, que se valida a hipótese de investigação proposta. Os resultados do presente estudo indicam a existência de uma relação positiva entre o valor funcional do UGC e a utilidade, ou seja, o valor funcional tem impacto positivo na utilidade percebida. Estes resultados estão alinhados com o estudo de Kim et al. (2012) e, como tal já seriam expectáveis.

Ao nível académico, este estudo contribui para um maior conhecimento acerca do uso do UGC, mais concretamente no YouTube, como fonte online de informações, inclusive no setor do turismo, sendo uma das plataformas a que os consumidores recorrem, enquanto turistas. Verificou-se, também, a adequabilidade desta relação para medir parte do 
fenómeno do UGC no YouTube. Adicionalmente, pode-se referir que o estudo confirmou que as escalas utilizadas para medir a relação são adequadas e consistentes.

Em termos empresariais, a análise da relação estabelecida neste estudo é relevante, tendo em consideração que, atualmente, já são muitos os visualizadores de conteúdo no YouTube em Portugal e a compreensão do comportamento dos consumidores para as empresas é fulcral, nomeadamente para as empresas que atuam no setor do turismo. Primeiramente, o estudo realça o facto do YouTube ser plataforma à qual os consumidores podem recorrer nas fases iniciais da sua consumer journey, quando utilizam fontes online na fase de procura de informações para tomar as suas decisões de compra, entre as quais decisões no contexto do turismo, tais como a escolha de hotéis, locais a visitar ou restaurantes a frequentar. Como tal, tendo em consideração toda a envolvente desse processo de decisão, torna-se essencial para as empresas, pelo menos, compreenderem o valor do UGC no YouTube e de que forma podem beneficiar deste tipo de conteúdo. O estudo revela que os consumidores valorizam conteúdo útil e credível. Neste sentido, os gestores e marketers devem ter em consideração estes aspetos, nomeadamente quando pretendem transmitir um determinado conteúdo. Para além disso, devem também considerar a utilização do formato de vídeo para comunicarem com o seu público-alvo. De uma forma geral, este estudo evidencia o papel e o valor do UGC no YouTube, sugerindo que as empresas que atuam no setor do turismo devem considerar a introdução das dinâmicas estabelecidas nesta plataforma nas suas estratégias de marketing, tornando-as mais eficazes e adequadas para atrair e reter os seus públicos-alvo. Por fim, tendo em atenção que o estudo se direciona para Portugal, é de destacar o contributo do mesmo, para os gestores e marketers de empresas que atuam no país, pois uma vez que existem poucos estudos na área, esta investigação vem trazer um maior conhecimento do fenómeno no mercado português.

Foram detetadas algumas limitações ao longo do desenvolvimento deste estudo, apresentadas em seguida. A utilização de uma amostra não aleatória e, como tal, não probabilística, não permite a generalização a toda a população dos resultados obtidos com o estudo. Para além do referido, tem-se o facto de ter sido apenas realizada uma análise de dados de natureza quantitativa, considerando-se ser interessante incluir também uma componente qualitativa, que tornaria o estudo mais completo; poderia ter sido realizada uma entrevista a um especialista na área ou a um focus group, de forma a obter novos inputs ao estudo ou confirmar os resultados obtidos.

Sugere-se alguns tópicos, cujo desenvolvimento poderá ter interesse em investigações futuras, relacionados com o tema do presente estudo. A caracterização do perfil dos consumidores de conteúdo gerado e partilhado no YouTube e uma compreensão mais aprofundada deste tipo de audiência poderia ser bastante relevante, assim como estudar de que forma as empresas/marcas podem beneficiar desse tipo de conteúdo divulgado na plataforma. Em relação ao modelo concetual utilizado neste estudo, este pode ser replicado atendendo a categorias de vídeo específicas ou de forma a averiguar possíveis diferenças entre faixas etárias e género; podem ser feitas pequenas alterações no modelo, inserindo variáveis como a qualidade do UGC (como estudado por Kim et al., 2012); ou pode ser aplicado ao conteúdo gerado e partilhado pelos utilizadores noutras redes sociais ou plataformas que reúnem comentários no âmbito turístico. 


\section{Referências}

Barreda, A. \& Bilgihan, A. (2013). An analysis of user-generated content for hotel experiences. Journal of Hospitality and Tourism Technology, 4(3), 263-280. doi.org/10.1108/JHTT-01-2013-0001

Cheong, H. J. \& Morrison, M. A. (2008). Consumers' reliance on product information and recommendations found in UGC. Journal of Interactive Advertising, 8(2), 38-49. doi.org/10.1080/15252019.2008.10722141

Constantinides, E. (2009, janeiro). Social media/web 2.0 as marketing parameter: An introduction. In Proceedings of the 8th International congress marketing trends (pp.15-17). Paris, France.

Constantinides, E. \& Fountain, S. J. (2008). Web 2.0: Conceptual foundations and marketing issues. Journal of Direct, Data and Digital Marketing Practice, 9(3), 231-244. doi.org/10.1057/palgrave.dddmp.4350098

Cormode, G. \& Krishnamurthy, B. (2008). Key differences between web 1.0 and web 2.0. First Monday, 13(6), 1-18. doi.org/10.5210/fm.v13i6.2125

Court, D., Elzinga, D., Mulder, S. \& Vetvik, O. J. (2009). The consumer decision journey. Disponível em https://www.mckinsey.com/business-functions/marketing-and-sales/our-insights/theconsumer-decision-journey

Cummins, S., Peltier, J. W., Schibrowsky, J. A. \& Nill, A. (2014). Consumer behavior in the online context. Journal of Research in Interactive Marketing, 8(3), 169-202. doi.org/10.1108/JRIM-04-2013-0019

Daugherty, T. (2008). Exploring consumer motivations for creating user-generated content. Journal of Interactive Advertising, 8(2), 16-25. doi.org/10.1080/15252019.2008.10722139

Davis, F., Bagozzi, R. \& Warshaw, P. (1989). User acceptance of computer technology: A comparison of two theoretical models. Management Science, 35(8), 982-1003. doi.org/10.1287/mnsc.35.8.982

Edelman, D. C. (2010). Branding in the digital age. Harvard Business Review, 88(12), 62-69.

Edelman, D. \& Singer, M. (2015a). Competing on customer journeys. Harvard Business Review, 93(11), 88-100.

Edelman, D. \& Singer, M. (2015b). The new consumer decision journey. Disponivel em http://valladaresdc.com/portfolio/diseno-publicitario-tenerife-amable/

GBSN Research. (2016). Inquérito - O uso das redes sociais. Disponível em http://www.gbsnresearch.com/wp-

content/uploads/2016/04/inquerito_sobre_o_uso_das_redes_sociais_-_gbsn_research.pdf

Google. (2015). Consumer barometer. Disponível em https://www.consumerbarometer.com

Google. (2016a). Consumer barometer. Disponível em https://www.consumerbarometer.com/en/graph-

builder/?question=M6\&filter=country:portugal

Google. (2016b). Curated insights. Disponível em https://www.consumerbarometer.com/en/insights/?countryCode=PT

Google. (2016c). How people meet their needs in I-want-to-know moments. Disponível em https://www.thinkwithgoogle.com/infographics/meet-needs-i-want-to-know-moments.html

Havas Media Group. (2016). Dados Digital [vídeo on youtube].

Kim, C., Jin, M-H., Kim, J. \& Shin, N. (2012). User perception of the quality, value, and utility of usergenerated content. Journal of Electronic Commerce Research, 13(4), 305-319.

Lecinski, J. (2011). Winning the zero moment of truth. Disponível em https://www.thinkwithgoogle.com/marketing-resources/micro-moments/2011-winning-zmotebook/

Levy, M. (2009). Web 2.0 implications on knowledge management. Journal of Knowledge Management, 13(1), 120-134. doi.org/10.1108/13673270910931215

Lieb, R., Owyang, J., Groopman, J. \& Silva, C. (2012). The converged media imperative: How brands must combine paid, owned, and earned media [slides]. Disponível em https://pt.slideshare.net/Altimeter/the-converged-media-imperative

Malhotra, N. K. \& Birks, D. F. (2006). Marketing research: An applied approach. Harlow: Prentice Hall

Marktest. (2016). Net panel - Audiências internet. Disponível em http://netpanel.marktest.pt/Dados/DadosTabela.aspx?tp=EvSemTopSites_UU\&idProd=1676

Marktest Consulting. (2015). Os portugueses e as redes sociais 2015. Disponível em http://www.marktest.com/wap/private/images/logos/Folheto_redes_sociais_2015.pdf 
Marktest Consulting. (2016). Os portugueses e as redes sociais 2016. Disponível em http://www.marktest.com/wap/private/images/Logos/Folheto_redes_sociais_2016.pdf

Marôco, J. (2014). Análise estatística com o SPSS Statistics (6. ${ }^{\mathrm{a}}$ ed.). Pêro Pinheiro: Report Number.

Mir, I. A. \& Rehman, K. U. (2013). Factors affecting consumer attitudes and intentions toward usergenerated product content on YouTube. Management \& Marketing, 8(4), 637-654.

O’Reilly, T. \& Battelle, J. (2009). Web squared: Web 2.0 five years on. Comunicação apresentada em Web 2.o Summit, San Francisco, CA.

Paek, H. J., Hove, T., Ju Jeong, H. \& Kim, M. (2011). Peer or expert? The persuasive impact of YouTube public service announcements producers. International Journal of Advertising, 30(1), 161-188. doi.org/10.2501/IJA-30-1-161-188

Pikas, B. \& Sorrentino, G. (2014). The effectiveness of online advertising: Consumer's perceptions of ads on Facebook, Twitter and YouTube. The Journal of Applied Business and Economics, 16(4), 70-81.

Pinho, J. C. M. R. \& Soares, A. M. (2011). Examining the technology acceptance model in the adoption of social networks. Journal of Research in Interactive Marketing, 5(2/3), 116-129. doi.org/10.1108/17505931111187767

Reino, S. \& Hay, B. (2011, junho). The use of YouTube as a tourism marketing tool. Comunicação apresentada na $47^{\text {th }}$ Annual International Conference of the Travel and Tourism Research Association, University of Massachusetts, Amherst.

Saunders, M., Lewis, P. \& Thornhill, A. (2009). Research methods for business students. Harlow: Prentice Hall.

Shao, G. (2009). Understanding the appeal of user-generated media: A uses and gratification perspective. Internet Research, 19(1), 7-25. doi.org/10.1108/10662240910927795

Sheth, J. N., Newman, B. I. \& Gross, B. L. (1991). Why we buy what we buy: A theory of consumption values. Journal of Business Research, 22(2), 159-170. doi.org/10.1016/0148-2963(91)90050-8

Smith, A. N., Fischer, E. \& Yongjian, C. (2012). How does brand-related user-generated content differ across YouTube, Facebook, and Twitter? Journal of Interactive Marketing, 26(2), 102-113. doi.org/10.1016/j.intmar.2012.01.002

Snelson, C. (2011). YouTube across the disciplines: A review of the literature. Journal of Online Learning and Teaching, 7(1), 159-169.

Wunsch-Vincent, S. \& Vickery, G. (2007). Participative web and user-created content: Web 2.0 wikis and social networking. Paris: OECD.

Ye, Q., Law, R., Gu, B. \& Chen, W. (2011). The influence of user-generated content on traveler behavior: An empirical investigation on the effects of e-word-of-mouth to hotel online bookings. Computers in Human Behavior, 27(2), 634-639. doi.org/10.1016/j.chb.2010.04.014

YouTube. (2016a). Acerca do YouTube. Disponível em https://www.YouTube.com/yt/about/pt-PT/

YouTube. (2016b). Sala de Imprensa. Disponível em https://www.YouTube.com/yt/press/pt-PT/

Zainuddin, N., Russell-Bennett, R. \& Previte, J. (2013). The value of health and wellbeing: An empirical model of value creation in social marketing. European Journal of Marketing, 47(9), 1504-1524. doi.org/10.1108/EJM-10-2011-0564

CAROLINA AFONSO é diretora de marketing na Konica Minolta e professora auxiliar convidada de Marketing \& Estratégia no ISEG. Tem mais de 12 anos de experiência na área das Tecnologias de Informação. É coordenadora executiva no ISEG/de alguns cursos de formação executiva: PósGraduação em Marketing Digital, em que leciona Marketing Digital e Social Media, da Pós-Graduação em Gestão da Sustentabilidade e do Curso Executivo em Social Media Management. Tem doutoramento Europeu em Marketing pela Universidad Complutense de Madrid, mestrado em Marketing, Pós-Graduação em Marketing Management pelo ISEG e licenciatura em Relações Internacionais pela Universidade Nova de Lisboa. É membro do centro de investigação Advance do ISEG. Endereço institucional: Departamento de Marketing, ISEG (Lisbon School of Economics \& Management), Rua do Quelhas, 6, Lisboa, 1200-781, Portugal. 
PATRÍCIA CEIA é analista de marketing na 360imprimir, onde trabalha na expansão e otimização de campanhas de search, em Google e Bing, em três mercados, Portugal, Espanha e Brasil. Tem cerca de dois anos de experiência em marketing digital. Tem mestrado em Marketing e licenciatura em Gestão pelo ISEG. Tem também formação em Google Adwords e Google Analytics. Endereço institucional: ISEG (Lisbon School of Economics \& Management), Rua do Quelhas, 6, Lisboa, 1200-781, Portugal.

Submetido em 20 setembro 2017

Aceite em 16 março 2018 


\section{Anexos}

\section{Anexo 1. Questionário}

\section{Introduçāo}

Estou a realizar um estudo com o objetivo de analisar o impacto do conteúdo gerado e partilhado pelos utilizadores no YouTube

$\square$ conteúdo gerado e partilhado pelos utilizadores é todo o tipo de conteúdo criado por qualque pessoa e partilhado na internet (neste caso, através do YouTube). São exemplos deste tipo de conteúdo vídeos comparativos de produtos (por exemplo: comparação entre smartphones), dicas de como os utilizar (por exemplo, produtos de maquilhagem]), testemunhos de utilização de serviços (por exemplo, opinião sobre restaurantes), entre outros. Este questionário tem uma duração média de 8 minutos.

Os dados recolhidos neste estudo são anónimos, confindenciais e serão tratados de forma agregada. Não há respostas certas nem erradas, pelo que peço a sua honestidade.

A sua colaboração é muito importante para o desenvolvimento do meu estudo.

$$
\text { 口brigada! }
$$

Questāo 1

Indique, por favor, quantas horas utiliza o YouTube por dia, em média, numa escala entre "Nunca" (1) e "Mais de 3 horas" (5). (Responda tendo em conta a sua opinião)

- Nunca o Menos de 1hora ontre 1e 2 horas o Entre 2 e 3 horas

$$
\text { Questāo } 2
$$

Indique, por favor, o seu grau de concordância com cada um dos seguintes motivos de utilização do YouTube, numa escala entre "Discordo totalmente" (1) e "Concordo totalmente" (7). (Responda tendo em conta a sua opinião) Utilizo o YouTube para:

Discordo Discordo em Discordo em Não concordo Concordo em Concordo em Concordo
totalmente

Criação de ligações interpessoais

Entretenimento.

Escape do quotidiano

Interação social

Partilha de informação

Passar o tempo

Procura de informação

Procura por companhia (quando estou sozinhola)

Questões profissionais

Visualização de vídeos com outras pessoas.

$\begin{array}{ll}\mathbf{0} & \mathbf{0} \\ \mathbf{0} & \mathbf{0} \\ \mathbf{0} & \mathbf{0} \\ \mathbf{0} & \mathbf{0} \\ \mathbf{0} & \mathbf{0} \\ \mathbf{0} & \mathbf{0} \\ \mathbf{0} & \mathbf{0} \\ \mathbf{0} & \mathbf{0} \\ \mathbf{0} & \mathbf{0} \\ \mathbf{0} & \mathbf{0}\end{array}$

$\mathbf{0}$
$\mathbf{0}$
$\mathbf{0}$
$\mathbf{0}$
$\mathbf{0}$
$\mathbf{0}$
$\mathbf{0}$
$\mathbf{0}$
$\mathbf{0}$

Questāo 3

Indique, por favor, a frequência com que vê cada uma das seguintes categorias de vídeo quando utiliza o Youtube, numa escala entre "Nunca" [1] e

\begin{tabular}{|c|c|c|c|c|c|c|c|}
\hline & Nunca & Raramente & Poucas vezes & $\begin{array}{c}\text { Algumas } \\
\text { vezes }\end{array}$ & Muitas vezes & $\begin{array}{l}\text { Quase } \\
\text { sempre }\end{array}$ & Sempre \\
\hline Animais & $\mathbf{0}$ & $\mathbf{0}$ & $\mathbf{o}$ & $\mathbf{0}$ & $\mathbf{0}$ & $\mathbf{0}$ & $\mathbf{0}$ \\
\hline Comédia & o & o & $\mathbf{o}$ & $\mathbf{o}$ & o & $\mathbf{o}$ & o \\
\hline Desporto & $\mathbf{o}$ & $\mathbf{o}$ & o & o & $\mathbf{o}$ & $\mathbf{o}$ & $\mathbf{o}$ \\
\hline Filmes e Animação & o & o & $\mathbf{0}$ & $\mathbf{0}$ & $\mathbf{0}$ & $\mathbf{0}$ & $\mathbf{0}$ \\
\hline Moda e Beleza & o & $\mathbf{o}$ & $\mathbf{o}$ & $\mathbf{0}$ & $\mathbf{o}$ & $\mathbf{o}$ & $\mathbf{o}$ \\
\hline Música & o & $\mathbf{0}$ & $\mathbf{o}$ & $\mathbf{0}$ & $\mathbf{0}$ & $\mathbf{0}$ & $\mathbf{o}$ \\
\hline Música & o & $\mathbf{0}$ & o & $\mathbf{0}$ & $\mathbf{0}$ & $\mathbf{0}$ & o \\
\hline Notícias e Política & $\mathbf{0}$ & $\mathbf{o}$ & o & o & $\mathbf{0}$ & $\mathbf{0}$ & $\mathbf{o}$ \\
\hline Pessoa influentes e Celebridades & $\mathbf{0}$ & $\mathbf{0}$ & $\mathbf{0}$ & $\mathbf{0}$ & $\mathbf{0}$ & $\mathbf{0}$ & $\mathbf{0}$ \\
\hline
\end{tabular}
"Sempre" (7). (Responda tendo em conta a sua opinião)

Indique, por favor, o seu grau de concordância com as seguintes afirmações, numa escala entre "Discordo totalmente" (1) e "Concordo totalmente" [7] (Responda tendo em conta a sua opinião)

De uma forma geral, o conteúdo gerado e partilhado pelos utilizadores no YouTube acerca de turismo:

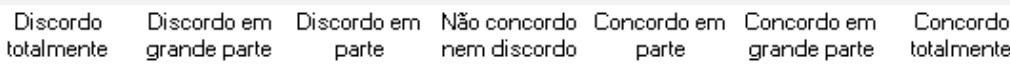

Tem uma qualidade consistente (ou seja, boa). É esplícito na informação a transmitir. Tem umn padrão de qualidade aceitável. Tem um bom desempenho (ou seja, cumpre o seu objetivo].

\begin{tabular}{ll} 
o & o \\
o & o \\
o & o \\
\hline & o
\end{tabular}

$\mathbf{0}$
$\mathbf{0}$
$\mathbf{0}$

o

$\mathbf{0}$

$\mathbf{0}$

o

o

o 


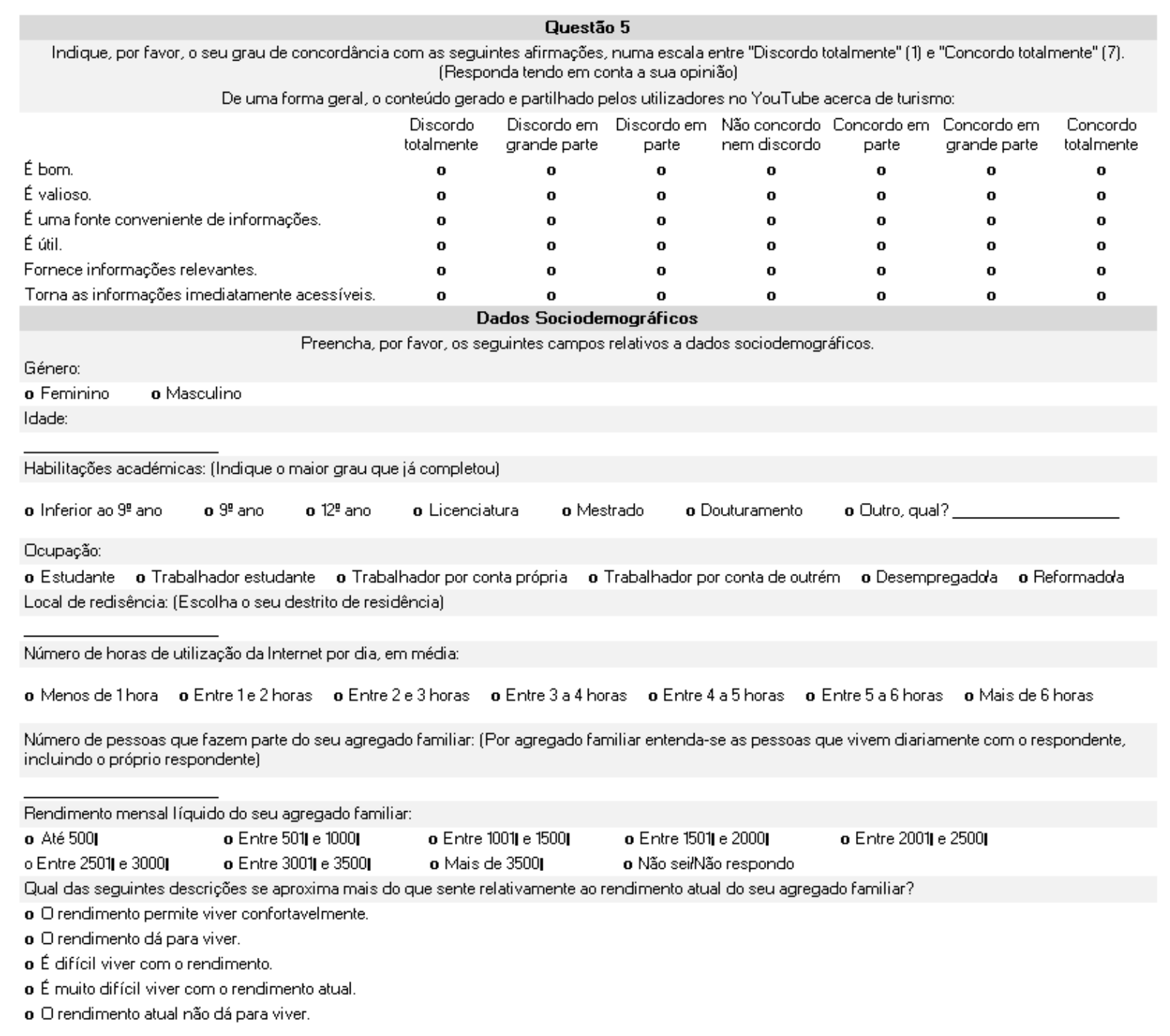


Anexo 2. Caracterização sociodemográfica da amostra

Tabela 3. Síntese dos dados sociodemográficos dos inquiridos
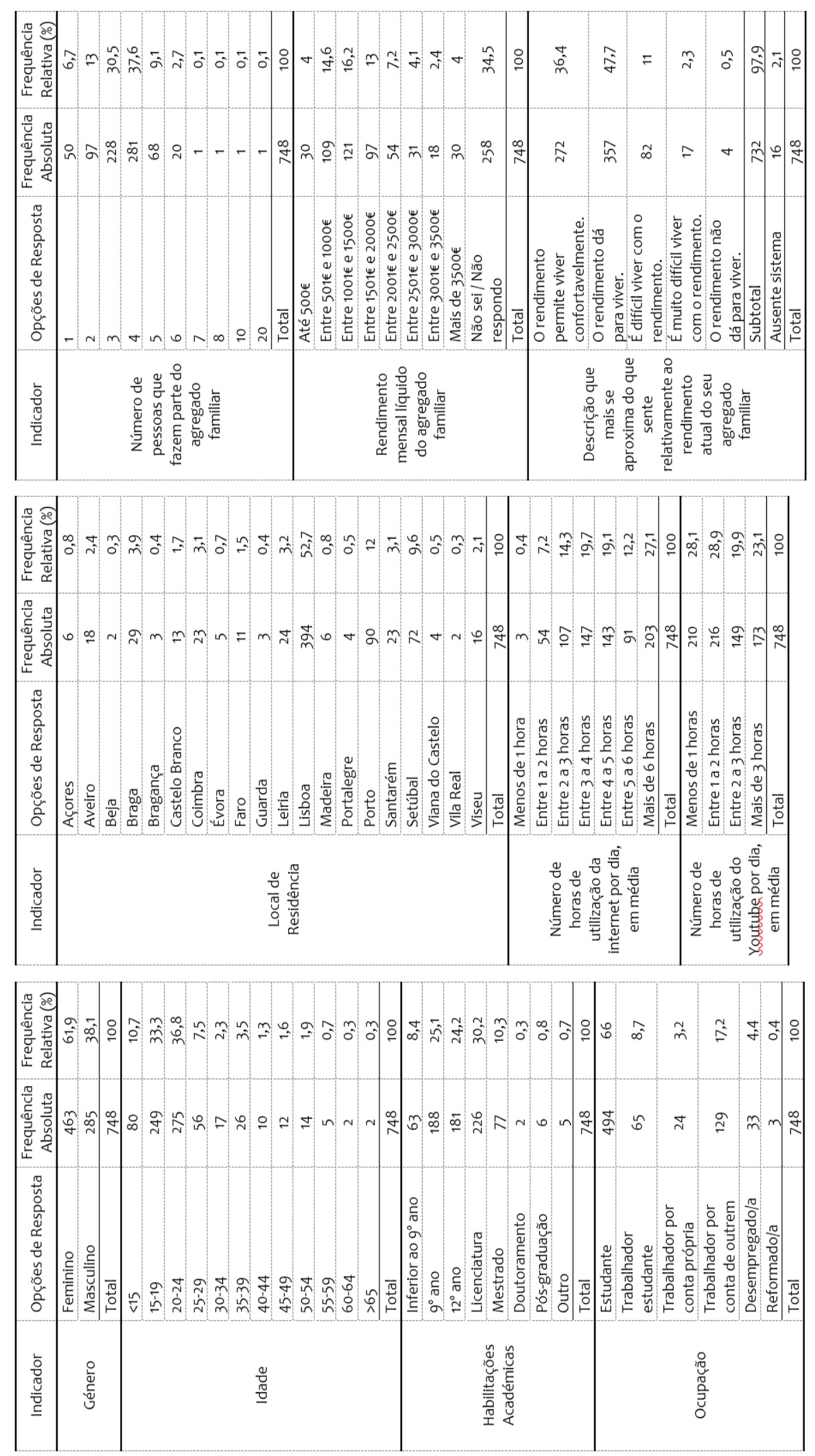
Anexo 3. Estatísticas descritivas das variáveis do estudo

Tabela 4. Síntese das estatísticas descritivas dos motivos de utilização do Youtube

\begin{tabular}{|c|c|c|c|c|c|c|c|c|}
\hline & & $\mathrm{N}$ & $\begin{array}{l}\text { Média } \\
(\overline{\mathrm{X}})\end{array}$ & $\begin{array}{l}\text { Mediana } \\
(\widetilde{\mathrm{X}})\end{array}$ & $\begin{array}{c}\text { Moda } \\
(\text { Mo) }\end{array}$ & $\begin{array}{c}\text { Desvio- } \\
\text { Padrão (S') }\end{array}$ & Mínimo & Máximo \\
\hline \multirow{10}{*}{ 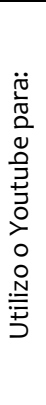 } & Criação de ligações interpessoais & 748 & 3,41 & 4 & 1 & 1,972 & 1 & 7 \\
\hline & Entretenimento & 748 & 6,54 & 7 & 7 & 0,839 & 1 & 7 \\
\hline & Escape do quotidiano & 748 & 5,27 & 5 & 7 & 1,569 & 1 & 7 \\
\hline & Interação social & 748 & 3,89 & 4 & 5 & 1,939 & 1 & 7 \\
\hline & Partilha de informação & 748 & 5,18 & 6 & 7 & 1,884 & 1 & 7 \\
\hline & Passar o tempo & 748 & 6,02 & 6 & 7 & 1,256 & 1 & 7 \\
\hline & Procura de informação & 748 & 5,8 & 6 & 7 & 1,312 & 1 & 7 \\
\hline & $\begin{array}{l}\text { Procura por companhia (quando } \\
\text { estou sozinho/a }\end{array}$ & 748 & 3,82 & 4 & 1 & 2,148 & 1 & 7 \\
\hline & Questões profissionais & 748 & 4,24 & 5 & 5 & 1,883 & 1 & 7 \\
\hline & $\begin{array}{c}\text { Visualização de vídeos com outras } \\
\text { pessoas }\end{array}$ & 748 & 5,07 & 5 & 5 & 1,615 & 1 & 7 \\
\hline
\end{tabular}

Codificação: 1 - Discordo Totalmente, 2 - Discordo em Grande Parte, 3 - Discordo em Parte, 4 - Não Concordo Nem Discordo, 5 - Concordo em Parte, 6 - Concordo em Grande Parte, 7 - Concordo Totalmente

Tabela 5. Síntese das estatísticas descritivas da frequência de visualização de categorias de vídeo

\begin{tabular}{|c|c|c|c|c|c|c|c|c|}
\hline & & $\mathrm{N}$ & $\begin{array}{c}\text { Média } \\
(\overline{\mathrm{X}})\end{array}$ & $\begin{array}{l}\text { Mediana } \\
(\widetilde{\mathrm{X}})\end{array}$ & $\begin{array}{c}\text { Moda } \\
\text { (Mo) }\end{array}$ & $\begin{array}{c}\text { Desvio- } \\
\text { Padrão (S') }\end{array}$ & Mínimo & Máximo \\
\hline \multirow{13}{*}{ 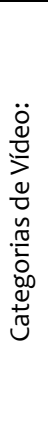 } & Animais & 748 & 2,78 & 2 & 2 & 1,395 & 1 & 7 \\
\hline & Comédia & 748 & 4,63 & 5 & 5 & 1,496 & 1 & 7 \\
\hline & Carros e Motos & 748 & 2,06 & 1 & 1 & 1,467 & 1 & 7 \\
\hline & Desporto & 748 & 2,96 & 3 & 1 & 1,75 & 1 & 7 \\
\hline & Filmes e Animação & 748 & 3,8 & 4 & 4 & 1,569 & 1 & 7 \\
\hline & How's to e DIY's (Do It Yourself) & 748 & 3,94 & 4 & 4 & 1,767 & 1 & 7 \\
\hline & Jogos & 748 & 3,32 & 3 & 1 & 2,148 & 1 & 7 \\
\hline & Moda e Beleza & 748 & 3,76 & 4 & 1 & 2,308 & 1 & 7 \\
\hline & Música & 748 & 5,96 & 6 & 7 & 1,182 & 1 & 7 \\
\hline & Notícias e Política & 748 & 2,77 & 2 & 1 & 1,574 & 1 & 7 \\
\hline & Pessoas influents e Celebridades & 748 & 3,21 & 3 & 2 & 1,704 & 1 & 7 \\
\hline & Tecnologia & 748 & 3,74 & 4 & 4 & 1,735 & 1 & 7 \\
\hline & Viagens e Lugares & 748 & 3,51 & 4 & 4 & 1,717 & 1 & 7 \\
\hline
\end{tabular}

Codificação: 1 - Nunca, 2 - Raramente, 3 - Poucas Vezes, 4 - Algumas Vezes, 5 - Muitas Vezes, 6 - Quase Sempre, 7 - Sempre

Tabela 6. Síntese das estatísticas descritivas dos índices sintéticos e dos itens que os compõem

\begin{tabular}{|c|c|c|c|c|c|c|c|c|c|c|}
\hline \multirow{2}{*}{ Variáveis } & \multirow{2}{*}{ Itens } & \multirow{2}{*}{$\mathrm{N}$} & \multicolumn{2}{|c|}{ Média $(\overline{\mathrm{X}})$} & \multirow{2}{*}{$\begin{array}{l}\text { Mediana } \\
(\widetilde{\mathrm{X}})\end{array}$} & \multirow{2}{*}{$\begin{array}{l}\text { Moda } \\
\text { (Mo) }\end{array}$} & \multicolumn{2}{|c|}{ Desvio-Padrão (S') } & \multirow{2}{*}{ Mínimo } & \multirow{2}{*}{ Máximo } \\
\hline & & & Item & Var. & & & Item & Var. & & \\
\hline \multirow{4}{*}{ Valor Funcional } & VF1 & 748 & 5,23 & \multirow{4}{*}{5,302} & 5,00 & 6 & 1,188 & \multirow{4}{*}{0,959} & 1 & 7 \\
\hline & VF2 & 748 & 5,15 & & 5,00 & 5 & 1,110 & & 1 & 7 \\
\hline & VF3 & 748 & 5,39 & & 5,00 & 5 & 1,084 & & 1 & 7 \\
\hline & VF4 & 748 & 5,44 & & 5,00 & 5 & 1,096 & & 1 & 7 \\
\hline \multirow{6}{*}{ Utilidade } & $\mathrm{U}_{1}$ & 748 & 5,47 & \multirow{6}{*}{5,38} & 5,00 & 5 & 1,130 & \multirow{6}{*}{0,921} & 1 & 7 \\
\hline & $\mathrm{U}_{2}$ & 748 & 4,83 & & 5,00 & 5 & 1,299 & & 1 & 7 \\
\hline & $U_{3}$ & 748 & 5,36 & & 5,00 & 5 & 1,210 & & 1 & 7 \\
\hline & $U_{4}$ & 748 & 5,60 & & 6,00 & 5 & 1,049 & & 1 & 7 \\
\hline & U5 & 748 & 5,37 & & 5,00 & 5 & 1,086 & & 1 & 7 \\
\hline & U6 & 748 & 5,65 & & 6,00 & 6 & 1,141 & & 1 & 7 \\
\hline
\end{tabular}

